\title{
Reação de genótipos de feijão guandu (Cajanus cajan (L.) Millspaugh) ao nematoide das galhas (Meloidogyne enterolobii)
}

\author{
Francisco José Carvalho Moreira ${ }^{1}$; Alexsandro Machado de Albuquerque ${ }^{2}$; Brena Kelly da Silva Almeida ${ }^{3}$; \\ Ivanderlete Marques de Souza ${ }^{4}$; Beatriz de Abreu Araújo ${ }^{5}$; Fernando Lisboa Guedes ${ }^{6}$
}

\begin{abstract}
${ }^{1}$ Doutorando em Biotecnologia RENORBIO/UFRN, prof. do Eixo Tecnológico de Recursos Naturais, Instituto Federal do Ceará, IFCE, Campus Sobral, Sobral - CE, Av. Dr. Guarani, 317 - Derby Clube. CEP: 62.042-030. Sobral - CE, Brasil ${ }^{2}$ Universidade Estadual Vale do Acaraú-UVA, Zootecnia, Sobral, CE; Av. da Universidade, 850 - Campus da Betânia. CEP: 62.040-370 - Sobral, CE., Brasil. ${ }^{3}$ Mestranda em Genética e Melhoramento de Plantas. Universidade Federal de Lavras. Programa de Pós-Graduação em Genética e Melhoramento de Plantas. Caixa Postal 3037 - CEP 37200-000 - Lavras, MG, Brasil. ${ }^{4}$ Universidade Estadual Vale do Acaraú - UVA. Programa de Pós-Graduação em Zootecnia UVA/ Embrapa Caprinos e Ovinos. Av. da Universidade, 850 - Campus da Betânia- Sobral, CE, Brasil. CEP: 62.040-370. ${ }^{5}$ Mestranda em Engenharia Agrícola, Universidade Federal do Ceará - UFC. Campus do PICI Departamento de Engenharia Agrícola - Bloco 804 Caixa Postal 12.168, CEP: 60450-760. Fortaleza, Ceará, Brasil. ${ }^{6}$ Pesquisador em Melhoramento Genético Vegetal. Embrapa Caprinos e Ovinos. Empresa Brasileira de Pesquisa Agropecuária (Embrapa). Fazenda Três Lagoas, Estrada Sobral/Groaíras, Km 4, Caixa Postal: 71, CEP: 62010-970 -Sobral, CE, Brasil. Autor para correspondência: Beatriz de Abreu Araújo (bia10_pcj@hotmail.com; beatrizdeabreuaraujo@gmail.com;)

Data de chegada: 24/10/2016. Aceito para publicação em: 12/09/2017.
\end{abstract}

$10.1590 / 0100-5405 / 170978$

\section{RESUMO}

Moreira, F.J.C.; Albuquerque, A.M.; Almeida, B.K.S.; Souza, I.M.; Beatriz de Abreu Araújo; Guedes, F.L. Reação de genótipos de feijão guandu (Cajanus cajan (L.) Millspaugh) ao nematoide das galhas (Meloidogyne enterolobii). Summa Phytopathologica, v.44, n.4, p.380-385, 2018.

A avaliação de genótipos de feijão guandu como antagônico, resistente ou tolerante ao nematoide das galhas (Meloidogyne enterolobii), pode elevar o potencial multiuso dessa leguminosa e verificar a reação de genótipos a esse fitopatógeno, considerado um dos mais agressivos do gênero, se faz necessário devido à sua ampla disseminação e escassez de controle no território nacional. Assim este trabalho teve por objetivo avaliar a reação de seis genótipos de feijão guandu (Cajanus cajan) ao patógeno $M$. enterolobii. A avaliação final das plantas ocorreu 80 dias após a inoculação dos fitopatógenos, na qual foram mensuradas variáveis de desenvolvimento das plantas: altura da planta, número de hastes, peso da matéria fresca da raiz e peso da matéria seca. Assim como também variáveis de desenvolvimento do nematoide $M$. enterolobii: número de galhas, índice de massas de ovos, fator de reprodução e redução do fator de reprodução. A hospedabilidade dos genótipos de feijão guandu foi avaliada por meio de cinco critérios classificatórios de distintos ajuizamentos. Para a produção de forragem, três genótipos foram moderadamente resistentes a M. enterolobii, FGSob/14-09, FGg59-95/14-37 e FG CAM/14-49. No entanto, os genótipos de feijão guandu não se enquadraram como plantas antagonistas, apresentando suscetibilidade ao fitopatógeno.

Palavras-chave: Fitopatógeno, Semiárido, Leguminosa Forrageira.

\section{ABSTRACT}

Moreira, F.J.C.; Albuquerque, A.M.; Almeida, B.K.S.; Souza, I.M.; Beatriz de Abreu Araújo; Guedes, F.L. Reaction of genotypes of pigeon pea (Cajanus cajan (L.) Millspaugh) to the root-knot nematode (Meloidogyne enterolobii). Summa Phytopathologica, v.44, n.4, p.380-385, 2018.

The eEvaluation of genotypes of pigeon pea genotypes as antagonistic, resistant or tolerant to the root-knot nematodes (Meloidogyne enterolobii), may increase the multipurpose potential of this legume, and verifying the reaction of genotypes reaction to this phytopathogen, considered one of the most aggressive pathogens of the genus, isf necessary due to its wide dissemination and scarcity of control in the national territory. Thus, the objective aim of this studywork was to evaluate the reaction of six genotypes of pigeon pea (Cajanus cajan) to the pathogen M. enterolobii. The final evaluation of the plants occurred 80 days after the inoculation of plant phytopathogens, and the following in which plant development variables were measured: plant height, stem number of stems, weight of root fresh root matter weight and weight of dry matter weight. As well as dDevelopmental variables of the nematode M. enterolobii were also evaluated: gall number of galls, egg mass index, reproduction factor and reproduction factor reduction. The host suitability genotypes of pigeon pea genotypes wasere evaluated based ony means of five classification criteria from different trials. For fodder the production of fodder, three genotypes were moderately resistant to M. enterolobii, FG Sob/14-09, FGg59-95/1437 and FGCAM/14-49. However, the genotypes of pigeon pea did not fit as antagonistic plants, presenting susceptibility to the phytopathogen.

Keywords: Phytopathogen, Semiarid, Forage Legume.

Desde o início dos projetos de irrigação para fruticultura no sudeste e nordeste brasileiro, os fitonematoides, principalmente os das galhas Meloidogyne spp., patógenos pouco importantes inicialmente, vem proporcionando aumento dos prejuízos, muitas vezes irreversíveis em algumas culturas frutíferas (3).

Devido à inexistência de métodos de controle eficientes, esta meloidoginose invariavelmente causa, em médio prazo, o declínio dos pomares com acentuada queda de produtividade, seguida de morte dos 
mesmos (17).

$\mathrm{Na}$ literatura encontram-se várias estratégias visando o controle desse fitopatógeno, nas áreas de resistência genética, manejo de pomares infestados, caracterização do patossistema e estimativa do impacto econômico e social do fitonematoide em algumas culturas, entre outras $(4,10,17,25)$.

Contudo, dentre os vários manejos testados para controle dos fitonematoides, há ainda alguns gargalos a serem superados, como por exemplo: 1) o controle químico de fitonematoides é normalmente caro para pequenos agricultores; 2) nematicidas nem sempre estão registrados para uso nas culturas objetos de exploração; 3) o período residual dos nematicidas no ambiente é, em geral, muito longo; 4) poucos vegetais cultivados apresentam resistência para a maioria das espécies de nematoides das galhas; 5) baixa disponibilidade de culturas antagônicas para utilização em rotação de culturas e combate ao nematoide $(6,14,27)$.

No sentido de identificar novas culturas antagônicas ao nematoide de galhas, especificamente ao Meloidogyne enterolobii, o objetivo deste trabalho foi avaliar a reação de seis genótipos de feijão guandu (Cajanus cajan) ao patógeno.

\section{MATERIAL E MÉTODOS}

O ensaio foi desenvolvido em casa de vegetação climatizada na Embrapa Caprinos e Ovinos, em Sobral, CE, com temperatura média de $30^{\circ} \mathrm{C}$ e com irrigação de $9,0 \mathrm{~mm}$ diários, distribuídos em seis irrigações de $1,5 \mathrm{~mm}$, por sistema intermitente. A área localiza-se a $3^{\circ} 45^{\prime} \mathrm{S}$ de latitude, longitude de $40^{\circ} 20^{\prime} \mathrm{W}$ e altitude de $80 \mathrm{~m}$, no período de março a julho de 2015. A multiplicação dos fitonematoides (inóculo) foi realizada em casa de vegetação no Instituto Federal de Educação, Ciência e Tecnologia do Ceará, Campus de Sobral - Ceará. A área localiza-se a $3^{\circ} 41^{\prime} \mathrm{S}$ de latitude, longitude de $40^{\circ} 20^{\prime} \mathrm{W}$.

Foram avaliados seis genótipos de feijão guandu pertencentes ao programa de melhoramento de forrageiras da Embrapa Caprinos e Ovinos: FGg66-95/14-38, FGRV/14-13, FGSob/14-09, FGg59-95/1437, FGJAN/14-01 e FGCAM/14-49. Os genótipos foram plantados em vasos de $10 \mathrm{~L}$ com solo argiloso tipo massapê.

O delineamento utilizado foi inteiramente casualizado (DIC) com parcelas de uma planta por vaso e 15 repetições. Para verificar a qualidade do inóculo, utilizaram-se duas testemunhas, tomate (Solanum lycopersicum) cultivar 'Santa Clara', uma com inóculo (positivo) e outra sem inóculo (negativo).

O inóculo de nematoide utilizado foi de população monoespecífica de $M$. enterolobii, confirmado pela técnica padrão de esterase, conforme sugerido por Carneiro \& Almeida (3) e multiplicada em tomateiro cv. Santa Clara, em casa de vegetação no IFCE - Campus Sobral. Os ovos e juvenis de segundo estádio $\left(\mathrm{J}_{2}\right)$ foram obtidos de suas raízes infestadas, seguindo as técnicas descritas por Hussey \& Barker (12) e Jenkins (13).

A inoculação foi realizada quando as plantas de feijão guandu apresentavam dois trifólios bem desenvolvidos. A suspensão com os ovos e $\mathrm{J}_{2}$ foi vertida em três orifícios a um centímetro de profundidade, feitos com um tubo de plástico comum a uma distância de 2,0 a 3,0 cm do colo de cada plântula.

Aos 80 dias da inoculação, realizou-se a avaliação final do ensaio, quando foram mensuradas as seguintes variáveis: número de galhas (NG; realizada por meio da contagem das galhas presentes nas raízes das plantas; número de ovos (NO); índice de massa de ovos (IMO); fator de reprodução (FR) e redução do fator de reprodução (RFR).

A hospedabilidade dos genótipos de feijão guandu foi avaliada por meio de cinco critérios classificatórios de distintos ajuizamentos. Assim, trabalhou-se com critérios de Dropkin \& Nelson (7), Seinhorst (23), Taylor \& Sasser (26), modificado por Hadisoeganda \& Sasser (11), Sasser et al. (21) e Moura \& Regis (16).

Os dados foram submetidos à análise de variância, conforme Ramalho et al. (18). Foram estimados os parâmetros genéticos como a herdabilidade no sentido amplo, o coeficiente de variação e a acurácia seletiva $(18,19)$.

As variáveis que apresentaram significância para o fator tratamento foram submetidas ao teste de agrupamento de médias Scott-Knott (22).

\section{RESULTADOS E DISCUSSÃO}

Todas as variáveis relacionadas ao desenvolvimento do nematoide M. enterolobii (NG, NO, NGgR, NOgR, FR e RFR) apresentaram diferenças significativas $(\mathrm{P}<0,01)$, o que indica que, entre os seis genótipos de feijão guandu avaliados, pelo menos um se diferiu quanto à reação ao nematóide (Tabela 1). Este fato evidenciou a ocorrência de variabilidade dos genótipos avaliados. Araújo Filho et al. (2), avaliando genótipos de feijão guandu quanto ao fator de reprodução do Meloidogyne javanica, também encontrou variabilidade entre os genótipos testados.

Observou-se que, para as variáveis NG e FR, os genótipos se dividiram em quatro grupos, em que os genótipos FGSob/14-09, FGg59-95/14-37 e FGCAM/14-49 apresentaram as menores médias do número de galhas, e o genótipo FGg66-95/14-38 apresentando o maior número de galhas (Tabela 2).

Tabela 1. Resumo da análise de variância (ANOVA), coeficiente de variação experimental (CV), acurácia seletiva $\left(\hat{\mathbf{r}}_{\hat{\mathrm{g}} \hat{\mathrm{g}}}\right)$ e herdabilidade no sentido amplo $\left(\mathrm{h}^{2}\right)$ das variáveis número de galhas $(\mathrm{NG})$, número de ovos $(\mathrm{NO})$, número de galhas por grama de raiz (NGgR), número de ovos por grama de raiz (NOgR), fator de redução (FR) e redução do fator de reprodução (RFR) de seis genótipos de feijão guandu inoculados com Meloidogyne enterolobii. Sobral-CE, 2015.

\begin{tabular}{|c|c|c|c|c|c|c|c|}
\hline $\begin{array}{l}\text { Fontes de } \\
\text { variação }\end{array}$ & G.L. & \multicolumn{6}{|c|}{ Quadrados Médios } \\
\hline CV (\%) & & 11,28 & 3,24 & 38,75 & 5,39 & 3,11 & 20,55 \\
\hline$\hat{\mathrm{r}}_{\hat{\mathrm{g}} \hat{\mathrm{g}}}$ & & 0,98 & 0,981 & 0,98 & 0,98 & 0,977 & 0,983 \\
\hline
\end{tabular}

significativo a $1 \%$, pelo teste $\mathrm{F}$. 
Tabela 2. Número de galhas (NG), número de ovos (NO), índice de massa de ovos (IMO), fator de redução (FR) e redução do fator de reprodução (RFR) de seis genótipos de feijão guandu inoculados com Meloidogyne enterolobii. Sobral-CE, 2015.

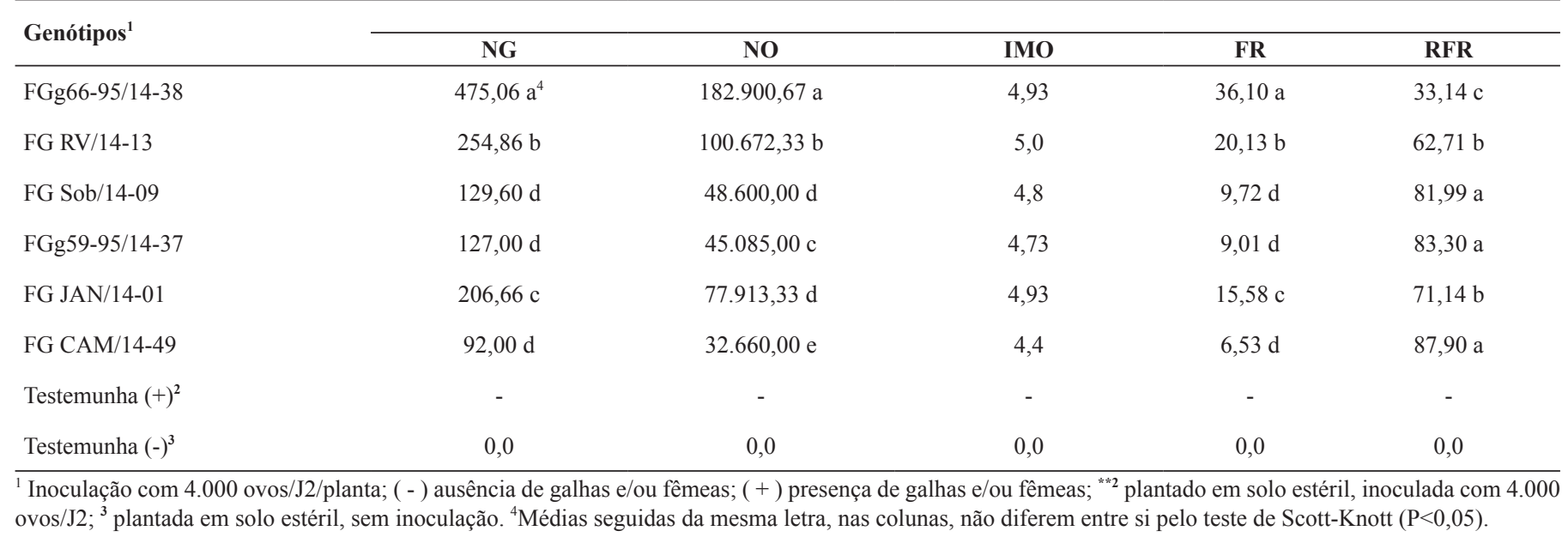

Araújo Filho et al. (2), avaliando genótipos de feijão guandu quanto ao fator de reprodução do $M$. javanica, caracterizou os genótipos FGg66-95/14-38 e FGg59-95/14-37 como resistentes para esta espécie de nematoide. Diante desses resultados, fica evidente que a caracterização de cultivares quanto à reação a um determinado nematoide não se estende para definir a reação das mesmas a outra espécie e/ou raça fisiológica desse patógeno.

Verificou-se que todas as variáveis relacionadas à reprodução e ao desenvolvimento do nematóide apresentaram valores de acurácia seletiva muito alta (Tabela 1). Variáveis com alta acurácia indicam pequenos desvios absolutos entre os valores genotípicos verdadeiros e aqueles estimados a partir das informações dos experimentos. Resende \& Duarte (19) destacaram a importância de se atingir acurácia seletiva ideal superior a 0,90 para inferência estatística segura.

Outro parâmetro genético importante é a herdabilidade, que informa o potencial hereditário do conjunto de genótipos avaliados em relação a cada variável avaliada. Nesse sentido, observou-se que todas as variáveis apresentaram alta herdabilidade, acima de $90 \%$, ou seja, a variação observada nos resultados desse experimento poderá ser herdada pelos descendentes dos genótipos avaliados.

Observamos que a testemunha positiva (tomate inoculado) foi altamente infectada pelo o inóculo, o que provocou a morte de todas as plantas da parcela.
A testemunha negativa (tomate não inoculado) não apresentou sintomas para o inóculo de $M$. enterolobii, além de não ter sido verificada a ocorrência de galhas nas raízes (Tabela 2). Este fato evidenciou que o solo utilizado no experimento não apresentava contaminação pelo fitopatógeno.

O genótipo FGCAM/14-49 foi o que apresentou menor valor do fator de reprodução (Tabela 2). Contudo, observou-se que a população do nematoide aumentou pelo menos seis vezes mais $(\mathrm{FR} \geq 6,0)$ que a população inicial no período avaliado, o que caracteriza os genótipos avaliados como não antagonistas a essa espécie de nematoide. Sharma et al. (24), em estudos de feijão guandu quanto a reação à M. javanica, encontraram resultados semelhantes.

A maioria das variáveis relacionadas ao desenvolvimento do hospedeiro (AP, NH, PMFR e PMSPA) apresentaram diferenças significativas $(\mathrm{P}<0,05)$ para os genótipos, exceto diâmetro do caule (Tabela 3). Estes resultados confirmaram, mais uma vez, a existência de variabilidade entre os genótipos de feijão guandu avaliados. Observouse precisão experimental fornecida pelo $\mathrm{CV}$, todas abaixo de $16 \%$. Contudo, algumas variáveis como DC e PMSPA apresentaram baixos valores de herdabilidade e de acurácia.

Apesar da alta infecção do nematoide $M$. enterolobii nos seis genótipos avaliados, o desenvolvimento vegetativo das plantas foi satisfatório, com alguns genótipos atingindo o estádio fenológico de

Tabela 3. Resumo da análise de variância (ANOVA), coeficiente de variação experimental (CV), herdabilidade no sentido amplo $\left(\mathrm{h}^{2}\right)$ e acurácia seletiva $\left(\hat{\mathrm{f}}_{\hat{\mathrm{g}} \mathrm{g}}\right)$ das variáveis de altura da planta $(\mathrm{AP})$, diâmetro do caule (DC), número de hastes $(\mathrm{NH})$, peso da meteria fresca da raiz (PMFR) e peso da matéria seca da parte aérea (PMSPA), de seis genótipos de feijão guandu inoculados com Meloidogyne enterolobii Yang \& Einsenback. Sobral-CE, 2015.

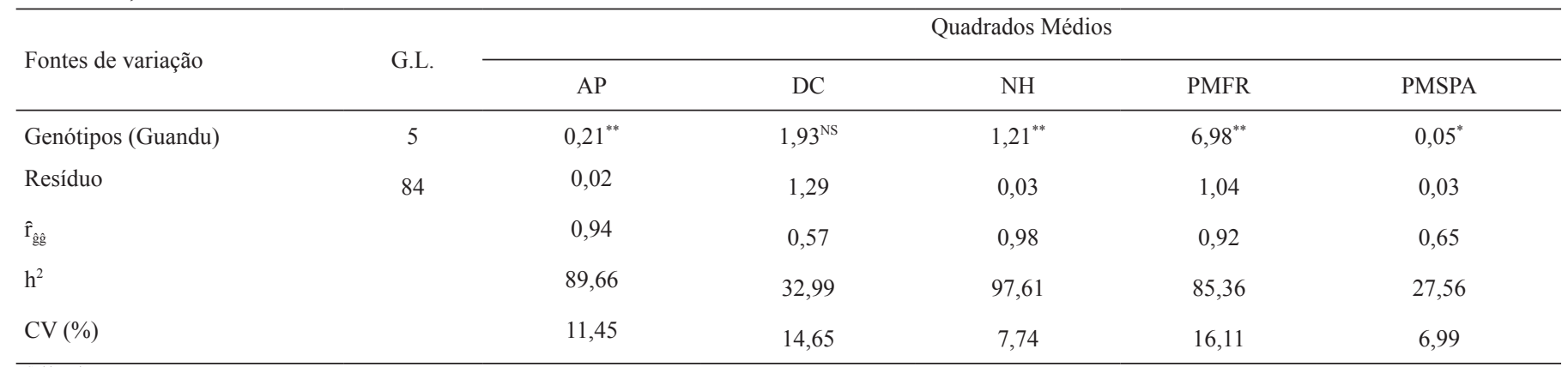

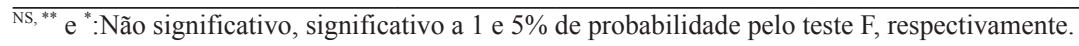


florescimento. Vale ressaltar que o período de 80 dias utilizado para avaliar o experimento foi suficiente para o nematoide completar pelo menos dois ciclos reprodutivos (20). Diante desses fatos, ficou evidente que o feijão guandu é uma planta hospedeira para o M. enterolobii, contudo, mostrou boa tolerância à presença do fitopatógeno, foi observado pelas variáveis do desenvolvimento do hospedeiro (Tabela 4). A existência de variabilidade para fator de reprodução indica que possam ser identificados genótipos antagônicos ao nematoide estudado.

Quanto à classificação dos seis genótipos de feijão guandu avaliados pelas cinco metodologias (Tabela 5), observou-se que, para três metodologias $\left(\mathrm{C}^{1}, \mathrm{C}^{2}\right.$ e $\left.\mathrm{C}^{5}\right)$, os genótipos foram classificados como suscetível e/ou bom hospedeiro para $M$. enterolobii. Diversos trabalhos na literatura reportaram a avaliação de genótipos de feijão guandu a várias espécies de Meloidogyne spp., os quais identificaram genótipos resistentes a algumas espécies $(2,21,24)$. Contudo, em nenhum desses trabalhos, os genótipos de feijão guandu foram avaliados a reação quanto a M. enterolobii ( $\sin$. M. mayaguensis), o qual apresenta alta agressividade em seu hospedeiro (15).

Duas metodologias $\left(\mathrm{C}^{3} \mathrm{e} \mathrm{C}^{4}\right)$ diferenciaram os genótipos quanto à classificação de comportamento à reação do M. enterolobii (Tabela 5). A metodologia $\mathrm{C}^{3}$ os classificou em suscetível ou intolerante, entretanto, essa classificação contém muita subjetividade, uma vez que não são definidos os parâmetros para avaliar o desenvolvimento do nematoide e do hospedeiro, sendo um método de pouco crédito.

Já a metodologia $C^{4}$, baseada na redução do fator de reprodução, é uma das mais utilizadas atualmente, pois amplia as possibilidades de avaliação da resistência dos genótipos. Por esse método foram identificados três genótipos moderadamente resistentes (FGSob/14-09, FGg59-95/14-37 e FGCAM/14-49), o que evidencia, mais uma vez, a existência de variabilidade quanto à reação ao $M$. enterolobii, apesar desses genótipos não atuarem como antagônicos ao fitopatógeno. A utilização de variedades resistentes constitui, juntamente com as práticas culturais, medida de grande relevância para o controle dos nematoides e tem como vantagens não oferecer riscos à saúde humana, ser de custo relativamente baixo e não poluir o ambiente. A identificação de genótipos de feijão guandu resistentes aos nematóides deve ser preocupação de importância nos programas de melhoramento dessa espécie.

Segundo Cruz et al. (5), o conhecimento da associação entre caracteres é de grande importância para os programas de melhoramento de qualquer espécie, principalmente quando a seleção em um deles apresenta dificuldades, devido à baixa herdabilidade ou problemas de medição. Nesse caso, pode ser recomendada a seleção indireta utilizando outro caráter que apresente alta herdabilidade e fácil avaliação, desde que ele esteja altamente correlacionado com aquele caráter de difícil seleção direta.

Por isso, foram analisadas as correlações entre as variáveis relacionadas com o desenvolvimento do nematoide e com as do hospedeiro (Tabela 6).

As variáveis NG e FR apresentaram correlações significativas com a maioria dos caracteres relacionadas com o desenvolvimento do hospedeiro. Dessa forma, plantas de feijão guandu com maior altura

Tabela 4. Altura da planta (AP), diâmetro do caule (DC), número de hastes (NH), peso da massa fresca da raiz (PMFR) e peso da massa seca da parte aérea (PMSPA) de seis genótipos de feijão guandu inoculados com Meloidogyne enterolobii. Sobral-CE, 2015.

\begin{tabular}{|c|c|c|c|c|c|}
\hline Genótipos & AP & DC & NH & PMFR & PMSPA \\
\hline FGg66-95/14-38 & $1,37 \mathrm{a}^{*}$ & $7,51 \mathrm{a}$ & $12,80 \mathrm{~b}$ & $53,02 \mathrm{a}$ & $27,46 \mathrm{a}$ \\
\hline FG Sob/14-09 & $1,45 \mathrm{a}$ & $7,91 \mathrm{a}$ & $22,00 \mathrm{a}$ & $34,46 \mathrm{~b}$ & $29,79 \mathrm{a}$ \\
\hline FGg59-95/14-37 & $1,33 \mathrm{a}$ & $7,47 \mathrm{a}$ & $12,33 \mathrm{~b}$ & $34,10 \mathrm{~b}$ & $27,37 \mathrm{a}$ \\
\hline FG CAM/14-49 & $1,19 \mathrm{~b}$ & $7,93 \mathrm{a}$ & $9,66 \mathrm{c}$ & $36,19 \mathrm{~b}$ & $23,27 \mathrm{a}$ \\
\hline
\end{tabular}

"Médias seguidas da mesma letra, na coluna, não diferem entre si pelo teste de $\operatorname{Scott-Knott~}(\mathrm{P}<0,05)$.

Tabela 5. Reação dos genótipos de feijão guandu a Meloidogyne enterolobii, de acordo com cinco metodologias distintas. Sobral-CE, 2015.

\begin{tabular}{|c|c|c|c|c|c|}
\hline \multirow[b]{2}{*}{ Genótipos } & \multicolumn{5}{|c|}{ Variáveis e comportamento } \\
\hline & $\mathrm{C}^{1}$ & $\mathrm{C}^{2}$ & $\mathrm{C}^{3}$ & $\mathrm{C}^{4}$ & $\mathrm{C}^{5}$ \\
\hline FGg66-95/14-38 & $\mathrm{S}$ & $\mathrm{BH}$ & $\mathrm{S}$ & $\mathrm{S}$ & $\mathrm{S}$ \\
\hline FG Sob/14-09 & $\mathrm{S}$ & $\mathrm{BH}$ & I & MR & $\mathrm{S}$ \\
\hline FG CAM/14-49 & $\mathrm{S}$ & $\mathrm{BH}$ & I & MR & $\mathrm{S}$ \\
\hline
\end{tabular}

$\mathrm{C}^{1}=$ Sasser et al. (1984), $\mathrm{R}=$ resistente e $\mathrm{S}=$ suscetível. $\mathrm{C}^{2}=$ Seinhorst (1967), $\mathrm{NH}=$ não hospedeira; $\mathrm{MH}=$ má hospedeira e BH = boa hospedeira.

$\mathrm{C}^{3}=$ Dropink \& Nelson (1960), $\mathrm{R}=$ resistente; $\mathrm{S}=$ suscetível; $\mathrm{T}=$ tolerante $\mathrm{I}=$ intolerante. $\mathrm{C}^{4}=\mathrm{Moura}(1997), \mathrm{I}=$ imune; $\mathrm{AR}=$ altamente resistente; MR = moderadamente resistente; $\mathrm{PR}=$ pouco resistente e $\mathrm{S}=$ suscetível. $\mathrm{C}^{5}=$ Taylor \& Sasser (1978), modificado por Hadisoeganda \& Sasser $(1982)$, AR = altamente resistente; $\mathrm{MR}=$ muito resistente; $\mathrm{LR}=$ levemente resistente; $\mathrm{Lr}=$ ligeiramente resistente e $\mathrm{S}=$ suscetível. 
Tabela 6. Correlações fenotípicas entre caracteres do feijão guandu inoculados com Meloidogyne enterolobii. Sobral-CE, 2015.

\begin{tabular}{|c|c|c|c|c|c|c|c|c|c|c|c|}
\hline Correl. & $\mathrm{NG}$ & NO & FR & RFR & $\mathrm{NGgR}$ & $\mathrm{NOgR}$ & $\mathrm{AP}$ & $\mathrm{DC}$ & $\mathrm{NH}$ & PMFR & PMSPA \\
\hline $\mathrm{NO}$ & & 1 & $-0,58^{*}$ & $0,77^{* *}$ & $-057^{*}$ & 0,56 & $-0,51$ & 0,52 & $-0,49$ & 0,47 & $-0,48$ \\
\hline FR & & & 1 & $-0,50$ & $0,83^{* *}$ & $-0,60^{*}$ & 0,48 & $-0,67^{*}$ & $0,63^{*}$ & $-0,80^{* * *}$ & $0,88^{* *}$ \\
\hline RFR & & & & 1 & $-0,50$ & 0,23 & $-0,45$ & 0,14 & $-0,43$ & 0,29 & $-0,42$ \\
\hline NOgR & & & & & & 1 & $-0,53$ & $0,74^{* *}$ & $-0,50$ & 0,53 & $-0,50$ \\
\hline $\mathrm{AP}$ & & & & & & & 1 & $-0,59^{*}$ & 0,40 & $-0,70^{* *}$ & 0,35 \\
\hline DC & & & & & & & & 1 & $-0,56$ & $0,85^{* *}$ & $-0,55$ \\
\hline $\mathrm{NH}$ & & & & & & & & & 1 & $-0,67^{*}$ & 0,55 \\
\hline
\end{tabular}

${ }^{* *} \mathrm{e}^{*}$ : significativo a 1,0 e $5,0 \%$ de probabilidade pelo teste $\mathrm{t}$, respectivamente.

(AP), menor diâmetro de caule (DC), maior número de hastes $(\mathrm{NH})$, menor peso de massa fresca da raiz (PMFR) e maior peso de massa seca da parte aérea (PMSPA) apresentaram a tendência ao maior de número de galhas (NG) e fator de reprodução (FR) do M. enterolobii. A maioria dos trabalhos de avaliação de hospedabilidade de feijão guandu foi com outras espécies de nematoides $(8,9,1)$, nos quais foram relatados altas reproduções de nematoides da espécie $R$. reniformis em cultivares de guandu, e não encontraram nenhuma relação de caracteres da planta com a presença do nematoide. No entanto, Thakar e Yadav (27) relataram que há uma variação entre cultivares de guandu quanto à resistência a R. reniformis, podendo este comportamento estar relacionado ao conteúdo de fenóis em cada genótipo, visto que variedades resistentes a R. reniformis apresentaram maiores teores de fenóis em seus tecidos do que variedades suscetíveis.

Os genótipos de feijão guandu avaliados apresentaram suscetibilidade e não se enquadraram como plantas antagonistas ao M. enterolobii.

Para a produção de forragem, três genótipos foram moderadamente resistentes a $M$. enterolobii, FGSob/14-09, FGg59-95/14-37 e FGCAM/14-49.

Plantas de feijão guandu (Cajanus cajan) com maior altura, menor diâmetro de caule, maior número de hastes, menor peso de massa fresca da raiz e maior peso de massa seca da parte aérea apresentaram o maior número de galhas e maior fator de reprodução do patógeno.

\section{REFERÊNCIAS}

1. Araújo Filho, J. V.; Inomoto, M. M.; Godoy, R.; Ferraz, L. C. C. B. Reação de linhagens de feijãoguandu a Rotylenchulus reniformis e Pratylenchus zeae. Nematologia Brasileira, Campinas, v. 34, n. 4, p. 204- 210, 2010.

2. Araújo Filho, J.V.; Inomoto, M.M.; Godoy, R.; Ferraz, L.C.C.B. Resistência de linhagens de feijão-guandu a Meloidogyne javanica. Nematologia Brasileira, Piracicaba, v.34, n.2, p.75-81, 2010.

3. Carneiro, R.M.D.G.; Almeida, M.R.A. Técnica de eletroforese usada no estudo de enzimas em nematoides das galhas para identificação de espécies. Nematologia Brasileira, Piracicaba, v.25. n.1, p.35-44, 2001.

4. Carneiro, R.M.D.G.; Cirotto, P.A.; Silva, D.B.; Carneiro, G.R. Resistance to Meloidogyne mayaguensis in Psidium spp. accessions and their grafting compatibility with P. guajava cv. Paluma. Fitopatologia Brasileira, Brasília. v.32, n.4, p. 281-284, 2007.

5. Cruz, C.D.; Regazzi, A.J.; Carneiro, P.C.S. Modelos biométricos aplicados ao melhoramento genético. Viçosa: Editora UFV, 2012, v.1, 514p.

6. Dropkin, V.H. Introduction to plant nematology. 2.ed. New York: John Wiley e Sons, 1989. 304p.

7. Dropkin, H.V.; Nelson, P.E. The histopathology of root-knot nematode infections in soybeans. Phytopathology, Salinas, v.50, n.6, p.442-447, 1960.

8. Gardiano, C. G.; Krzyzanowski, A. A.; Saab, O. J. G. A. Hospedabilidade de plantas melhoradoras de solo à Rotylenchulus reniformis. Linford e Oliveira (1940). Arquivos do Instituto Biológico, São Paulo, v. 79, n. 2, p. 313-317, 2012.

9. Gardiano, Cristiane Gonçalves ; Krzyzanowski, Alaide Aparecida ; Abi Saab, Otavio Jorge Grigoli . Eficiência de espécies de adubos verdes sobre a população do nematoide reniforme. Semina. Ciências Agrárias (Impresso), v. 35, p. 719-726, 2014.

10. Gomes, V.M.; Souza, R.M; Melarato, M.; Dolinski, C. Caracterização do Estado nutricional de goiabeiras em declínio parasitadas por Meloidogyne mayaguensis. Nematologia Brasileira, Piracicaba, v.32, p.154-160, 2008.

11. Hadisoeganda, W.W.; Sasser, J.N. Resistence of tomato, bean, southern pea and garden pea cultivars to root-knot nematodes based on host suitability. Plant Disease. Saint Paul, v.66, n.2, p.145-149, 1982.

12. Hussey, R.S.; Barker, K.R. A comparison of methods of collecting inocula for Meloidogyne spp., including a new technique. Plant Disease Reporter, Washington, v.57, n.12, p.1025-1028, 1973.

13. Jenkins, W.R. A rapid centrifugal-flotation technique for separating nematodes from soil. Plant Disease Reporter, Washington, v.48, n. 41, p.692, 1964.

14. Montalvo, A.E.; Esnard, J. Reaction of tem cultivars of watermelon (Citrullus lanatus) to a Puerto Rican population of Meloidogyne incognita. Journal of Nematology. Hanover, v.26, n.4, p.640-643, 1994

15. Moreira, F.J.C.; Silva, M.C.B.; Rodrigues, A.A.; Tavares, M.K.N. Alternative control of root-knot nematodes (Meloidogyne javanica and M. enterolobii) using antagonists. International Journal of Agronomy and Agricultural Research, v.7, n.2, p.121-129, 2015.

16. Moura, R.M.; Régis, E.M.O. Reações de cultivares de feijoeiro comum (Phaseolus vulgaris) em relação ao parasitismo de Meloidogyne javanica e Meloidogyne incognita (Nematoda: Heteroderidae). Nematologia Brasileira, Piracicaba, v.11, p.215-225, 1987.

17. Pereira, F.O.M.; Souza, R.M.; Souza, P.M.; Dolinski, C.; Santos. G.K. Estimativa do impacto econômico e social direto de Meloidogyne enterolobii na cultura da goiaba no Brasil. Nematologia Brasileira, Piracicaba, v.33, p.176-181, 2009

18. Ramalho, M.A.P.; Ferreira, D.F.; Oliveira, A.C. Experimentação em 
genética e melhoramento de plantas. 3 ed. Lavras: UFLA. 2012. 326p.

19. Resende, M.D.V.; Duarte, J.B. Precisão e controle de qualidade em experimentos de avaliação de cultivares. Pesquisa Agropecuária Tropical, Goiânia, v.37, n.3, p.182-194, 2007.

20. Rosa, J.M.O.; Westerich, J.N.; Wilcken, S.R.S. Reprodução de Meloidogyne enterolobii em olerícolas e plantas utilizadas na adubação verde. Revista Ciência Agronômica, Fortaleza, v.46, n.4, p.826-835, 2015.

21. Sasser, J.N.; C.C. Carter; K.M. Hartman. Standardization of host suitability studies and reporting of resistance to root-knot nematodes. Raleigh: North Caroline State University, 1984. 7p.

22. Scott, A.J.; Knott, M.A.A. Cluster analysis method for grouping means in the analysis of variance. Biometrics, Raleigh, v.30, n.3, p.507-512, 1974.

23. Seinhorst, J.W. The relationships between population increase and population density in plant parasitic nematodes. Nematologica, Leida, v.13, p.157-171, 1967.

24. Sharma, S.B.; Smith, D.H.; McDonald, D. Nematode constraints of chickpea and pigeonpea production in the semi-arid tropics. Plant Disease, Saint Paul, v.75, p.868-874, 1992.
25. Souza, M.M.; Pereira, T.N.S.; Dias, A.J.B.; Ribeiro, B. .; Viana, A.P. Structural, Hystochemical and Cytochemical Characteristics of the Stigma and Style in Passiflora edulis f. flavicarpa (Passifloraceae). Brazilian Archives of Biology and Technology, Curitiba, v.49, n.1, p.93-98, 2006.

26. Taylor, A.L.; Sasser, J.N. Biology, identification and control of root-knot nematodes (Meloidogyne species). Raleigh: North Caroline State University. 1978. 111p.

27. Thakar, N. A.; Yadav, B. S. Role of total phenols in pigeonpea resistance to reniform nematode. Indian Journal of Nematology, Lakeland, v. 16 , n. 2, p. 261-263, 1986.

28. Torres, G. R. C. Nematofauna associada ao meloeiro em uma área de cultivo no Rio Grande do Norte, reação de genótipos de cucurbitáceas a Rotylenchulus reniformis, caracterização e sobrevivência do parasito. Anais da Academia Pernambucana de Ciência Agronômica, Recife, v.4, p.162-184, 2007. 